\title{
CMEARTICLE
}

\section{Clinics in diagnostic imaging (173)}

Vishal Gaikwad ${ }^{1}$, MBBS, DNB, Ashish Chawla ${ }^{1}$, DABR, Tze Chwan $\underline{L i m}^{1}$, MBBS, FRCR, Wilfred CG $\underline{\text { Peh }}^{1}$, FRCP, FRCR

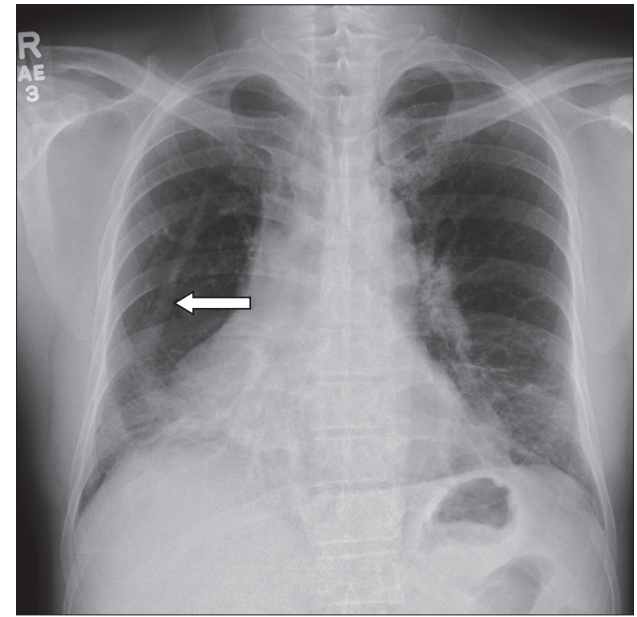

Fig. 1 Posteroanterior radiograph of the chest.
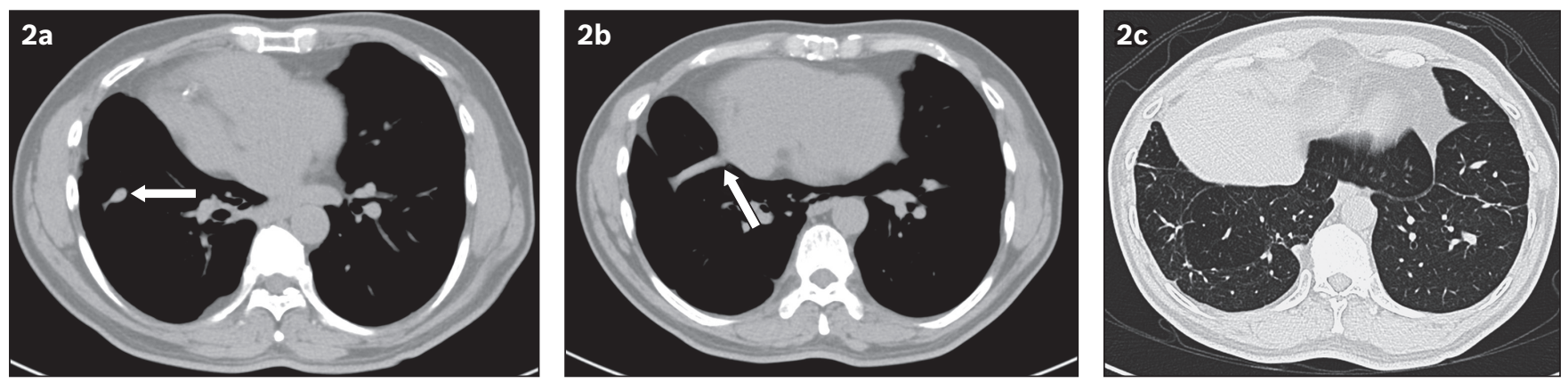

Fig. 2 Axial CT images of the mid and lower thorax on (a \& b) soft tissue and (c) lung windows.

\section{CASE PRESENTATION}

A 56-year-old Chinese man had chest radiography performed as part of a routine work-up for a recent onset of vestibular neuronitis. He presented with severe giddiness and positional vertigo. There was no history of cough, fever or breathlessness. The clinical examination revealed no rales, crepitation or wheezing. There was no previous history of chest infection. The pulse rate, blood pressure and saturation of peripheral oxygen $\left(\mathrm{SpO}_{2}\right)$ were normal. What do the frontal chest radiograph (Fig. 1) and subsequent non-contrast computed tomography (CT) images of the thorax (Figs. 2a-c) show? 


\section{IMAGE INTERPRETATION}

The chest radiograph shows a vertical curvilinear opacity parallel to the right heart border, which gradually increases in width along its caudal course before merging with the right lower cardiomediastinal silhouette, producing the scimitar sign (arrow, Fig. 1). There is right lower zone hypoplasia with a deviation of the heart shadow to the right (consistent with mild dextroposition) and indistinctness of the right heart border. The left lung appears unremarkable. Non-contrast CT shows the anomalous vascular channel coursing caudally (arrow, Fig. 2a), turning medially and ultimately draining into the supradiaphragmatic inferior vena cava (arrow, Fig. 2b). A horseshoe configuration of the lung is identified, in which the medial segment of the middle lobe is seen to cross the midline and fuse with the left lung (Fig. 2c). The crossing parenchymal tissue lies posterior to the pericardial sac but anterior to the oesophagus and aorta. The scimitar vein, anomalous course of the middle lobe bronchovascular bundle and pleural line of fusion are better delineated on the reconstructed coronal images (Figs. 3a-c). No anomalous vascular channel was seen in the left lung. No air trapping, which would have indicated underlying bronchial stenosis, was discernible in the displaced right lung. No overt sign of pulmonary arterial hypertension, such as an enlarged pulmonary trunk and increased main pulmonary artery to ascending aorta ratio, was observed. Cardiac chamber enlargement or any obvious cardiac anomaly was not appreciated within the limits of the unenhanced CT images.

\section{DIAGNOSIS}

Scimitar syndrome with horseshoe lung.

\section{CLINICAL COURSE}

The patient responded to treatment for the presenting symptoms of giddiness and positional vertigo. No further assessment of scimitar syndrome or associated cardiac anomaly was pursued.

\section{DISCUSSION}

Scimitar syndrome is characterised by an anomalous pulmonary venous return, in which the venous channel from the lung drains into the systemic vein rather than the left atrium. It falls under the broad category of congenital pulmonary venolobar syndrome..$^{(1)}$ This complex pulmonary malformation has an incidence rate of 1-3 per 100,000 and a 2:1 female preponderance. The anomalous pulmonary vein typically drains the lung partially into the systemic circulation, which is usually the infradiaphragmatic inferior vena cava. Other rare drainage sites are the right atrium, superior vena cava, portal vein, hepatic vein and azygous vein..$^{(2)}$ This drainage pattern leads to left-to-right shunting which, if significant, can result in the development of pulmonary arterial hypertension. Although the anomaly is usually right-sided, left-sided scimitar syndrome ${ }^{(3)}$ and cases of bilateral scimitar syndrome ${ }^{(4)}$ have been reported. Two forms have been described based on the age of presentation: infantile and adult forms. The infantile form is more symptomatic and commonly associated with congenital cardiac defects such as atrial septal defect, persistent left superior vena cava and vascular rings. The adult form is less severe, mostly asymptomatic and has a relatively good prognosis.

Clinically, the scimitar anomaly can be asymptomatic and detected incidentally on imaging, or present with severe pulmonary hypertension (typically in the infantile form with associated congenital cardiac defects). Radiographically, a clue to the presence of an abnormal pulmonary venous return is the demonstration of a curvilinear opacity in the lung that starts from the hilum, has a parallel course to the heart border and widens inferiorly toward the diaphragm, such that it resembles a scimitar (i.e. Turkish sword) (Fig. 1). Other radiographic signs include right lung hypoplasia, dextroposition, indistinctness of the right heart border and decreased right pulmonary vascularity.

The scimitar sign, which was once thought to be diagnostic of scimitar syndrome, can be mimicked by other benign vascular anomalies such as a meandering pulmonary vein and an anomalous single unilateral pulmonary vein. ${ }^{(5)} \mathrm{A}$ meandering pulmonary vein occurs when one of the pulmonary veins draining into the left atrium has an anomalous pulmonary course. Another mimic of the scimitar sign is an anomalous single unilateral pulmonary vein, which is a single pulmonary trunk draining all the bronchopulmonary segments to the left atrium. It is clinically important to distinguish these benign entities from scimitar syndrome to avoid unnecessary intervention and imaging. As middle lobe collapse, pulmonary sequestration, pulmonary venous varix and intrapulmonary collaterals secondary to obstruction of the main pulmonary vein can also mimic the scimitar sign, this imaging sign may be suggestive but is not diagnostic of scimitar syndrome. ${ }^{(6)}$ The radiographic opacity of middle lobe collapse often has a more horizontal orientation compared to the scimitar vein, which courses in a vertical and curvilinear fashion (Fig. 4a). Right middle lobe collapse is limited by the major and minor fissures, and shows fissural displacement.

These findings can be confirmed by lateral chest radiography or CT (Figs. 4b \& c). However, it has to be stressed that the classical radiographic signs of scimitar syndrome may not be present in all cases and that helpful secondary signs, such as altered lung anatomy following lung hypoplasia and cardiac dextroposition, may obscure the demonstration of the scimitar vein. CT is often the next step in evaluating the spatial anatomy of the scimitar vein, the drainage site of the vein, pulmonary hypertension, aortopulmonary collaterals, anomalous arterial supply from the aorta to the lung, and pulmonary sequestration. Magnetic resonance (MR) imaging is an excellent non-ionising, non-invasive method that allows detailed evaluation of the pulmonary veins, assessment of ventricular volume, and ventricular function and quantification of left-to-right shunt fraction. Cardiac catheterisation and angiography are a definitive means of establishing the diagnosis, but should only be sought when endovascular intervention is being contemplated.

The diagnostic value of angiography is limited in cases of pulmonary atresia or high-grade stenosis; in such cases, MR imaging and $\mathrm{CT}$ are helpful alternatives to evaluate extracardiac venous and arterial anatomy. ${ }^{(7)}$ Preoperative imaging of scimitar syndrome should aim to evaluate: (a) whether the pulmonary 

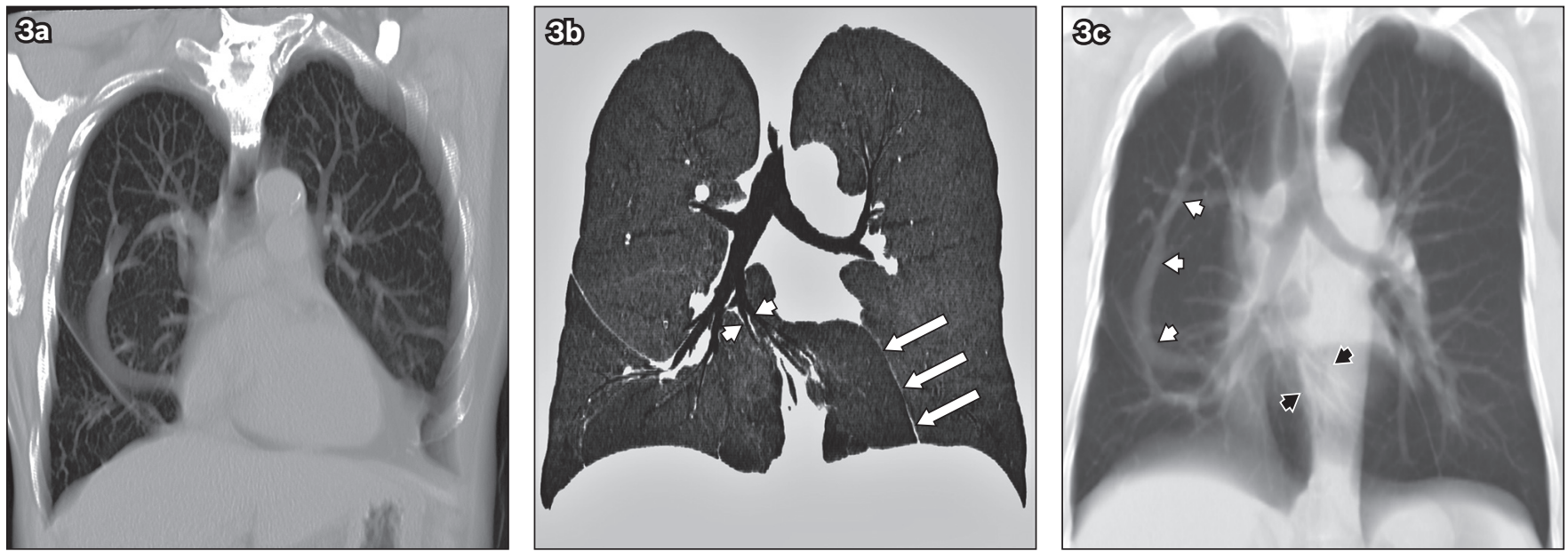

Fig. 3 Anatomical configuration of a scimitar vein and horseshoe lung. (a) Reconstructed oblique coronal CT image shows partial anomalous venous return in the entire extent and drainage into the supradiaphragmatic inferior vena cava. (b) Coronal CT minimal intensity projection image shows the isthmus of horseshoe lung, altered course of the right middle lobe segmental bronchi (white arrowheads) and pleural line of fusion (white arrows). (c) Thick reconstructed coronal CT image shows the scimitar vein in its entirety (white arrowheads) and altered course of the right middle lobe bronchovascular bundle (black arrowheads).
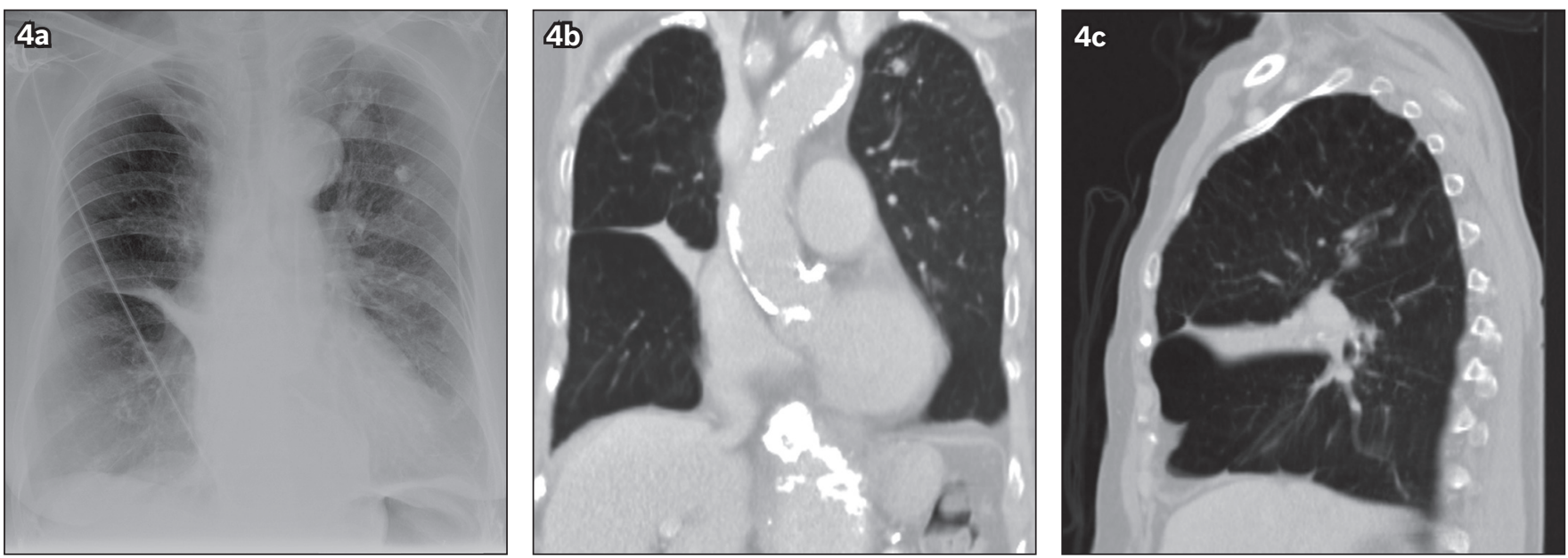

Fig. 4 Imaging mimics of the scimitar sign. (a) Posteroanterior chest radiograph shows oblique tubular opacity in the right middle zone, which merges with the right heart border and resembles the scimitar sign. ( $b$ \& c) Coronal and sagittal minimal intensity projection CT images show triangular opacity in the right middle zone due to right middle lobe collapse.

anomalous return is total or partial; (b) presence of pulmonary hypertension; (c) left-to-right shunt fraction; (d) presence of associated congenital cardiac defects; (e) presence of aortopulmonary collaterals; and (f) presence of anomalous arterial supply to the hypoplastic lung. Surgical repair is warranted if the shunt fraction (Qp:Qs) is > 2. The surgical options of direct reimplantation of the scimitar vein and intracardiac baffle repair are generally safe and efficient. However, higher mortality and complication rates are observed in infants and patients with associated pulmonary arterial hypertension. ${ }^{(8)}$

Horseshoe lung, a term that was first used by Spencer in 1962 , is a rare lung malformation occurring in almost $80 \%$ of patients with scimitar syndrome. ${ }^{(9,10)}$ In this rare lung anomaly, the posterobasal segments of both lungs usually show fusion in the midline through an isthmus of normal lung tissue. A parietal pleural defect allows for direct or indirect adherence of lung parenchyma through the intervening visceral pleura. ${ }^{(11)}$ The isthmus of normal lung tissue lies posterior to the pericardial sac but anterior to both the oesophagus and aorta. Unilateral pulmonary hypoplasia is a constant feature of horseshoe lung and the bronchovascular supply to the isthmus is usually from the hypoplastic lung. Frank et al described the radiographic signs of horseshoe lung on chest radiographs; these included the presence of a well-defined area similar to an inferior accessory lobe and a fissure medial to the left lung. ${ }^{(9)}$ This fissure corresponds to a pleural line separating the displaced right lung and the adjoining left lung, and is a consistent finding of horseshoe lung on CT. ${ }^{(12)}$ The fused posterobasal segments of the right and left lungs may show imaging features of air trapping, suggesting possible stenosis of anomalous bronchus of the isthmus. ${ }^{(13)}$ The functional importance of excluding a stenosed anomalous bronchus lies in the fact that impaired bronchial clearance results in recurrent pneumonia in the horseshoe lung segment, necessitating pneumonectomy or lobectomy. In the absence of other malformations, the prognosis of scimitar syndrome with horseshoe lung primarily depends on the pulmonary arterial pressure. A large shunt fraction is associated with a high risk of developing pulmonary arterial hypertension, although the patient 
is usually symptomatic in infancy or childhood. In cases of mild to absent left-to-right shunt, the patient is usually asymptomatic due to near-normal pulmonary arterial pressure and the absence of pulmonary hypertension. Hence, it can be deduced that an isolated association with horseshoe lung has little bearing on the prognosis of scimitar syndrome. ${ }^{(12)}$

In conclusion, scimitar syndrome and horseshoe lung have characteristic imaging features. Up to $80 \%$ of reported cases of horseshoe lung have been seen in association with scimitar syndrome. Therefore, it is essential for the radiologist to be aware of this rare entity when evaluating a patient with scimitar syndrome. Multiplanar cross-sectional imaging studies aid in the assessment of underlying anatomy and differentiation among various imaging mimics.

ABSTRACT A 56-year-old Chinese man presented with giddiness and vertigo. Subsequent chest radiography showed the classic scimitar sign of an abnormal pulmonary venous return. Further evaluation with noncontrast computed tomography substantiated the finding of a partial anomalous venous drainage pattern and identified an associated rare lung anomaly, horseshoe lung. The imaging findings of scimitar syndrome and its association with horseshoe lung are reviewed.

Keywords: horseshoe lung, isthmus, meandering pulmonary vein, pulmonary venous return, scimitar syndrome

\section{REFERENCES}

1. Woodring JH, Howard TA, Kanga JF. Congenital pulmonary venolobar syndrome revisited. Radiographics 1994; 14:349-69.

2. Bratincsák A, Rao RP, El-Said HG. Unusual variant of a rare constellation: a left-sided scimitar syndrome with connection to the azygos vein. Congenit Heart Dis 2010; 5:174-7.

3. Juraszek AL, Cohn H, Van Praagh R, Van Praagh S. Isolated left-sided scimitar vein connecting all left pulmonary veins to the right inferior vena cava. Pediatr Cardiol 2005; 26:846-7.

4. Kabbani M, Haider N, Abu-Sulaiman R. Bilateral scimitar syndrome. Cardiol Young 2004; 14:447-9.

5. Lee ML. Isolated and complex scimitar vein anomalies and their differentiation from the meandering right pulmonary vein. Yonsei Med J 2007; 48:973-80.

6. Nazarian J, Kanne JP, Rajiah P. Scimitar sign. J Thorac Imaging 2013; 28:W61.

7. El-Medany S, El-Noueam K, Sakr A. Scimitar syndrome: MDCT imaging revisited. Egyptian J Radiol Nucl Med 2011; 42:381-7.

8. Huddleston CB, Exil V, Canter CE, Mendeloff EN. Scimitar syndrome presenting in infancy. Ann Thorac Surg 1999; 67:154-9, discussion 160.

9. Frank JL, Poole CA, Rosas G. Horseshoe lung: clinical, pathologic, and radiologic features and a new plain film finding. AJR Am J Roentgenol 1986; 146:217-26.

10. Takedo K, Kato N, Nakagawa T, Aoki K, Matsuda A. Horseshoe lung without respiratory distress. Pediatr Radiol 1990; 20:604.

11. Hawass ND, Badawi MG, al-Muzrakchi AM, et al. Horseshoe lung: differential diagnosis. Pediatr Radiol 1990; 20:580-4.

12. Dupuis C, Rémy J, Rémy-Jardin M, et al. The "horseshoe" lung: six new cases. Pediatr Pulmonol 1994; 17:124-30.

13. Takahashi M, Murata K, Yamori M, et al. Horseshoe lung: demonstration by electron-beam CT. Br J Radiol 1997; 70:964-6. 


\section{SINGAPORE MEDICAL COUNCIL CATEGORY 3B CME PROGRAMME} (Code SMJ 201701B)

Question 1. Regarding scimitar syndrome:

(a) It has a female preponderance.

(b) The most common pattern of lung drainage by the scimitar vein is partial lung drainage.

(c) It shows left-to-right shunting which, if large enough, can result in pulmonary hypertension.

(d) It is commonly associated with ventricular septal defect, right-sided aortic arch and pulmonic stenosis.

Question 2. Regarding the scimitar sign:

(a) It refers to a vertical curvilinear opacity parallel to the right heart border, which gradually increases in width along its caudal course before merging with the right lower cardiomediastinal silhouette.

(b) It is specific for scimitar syndrome.

(c) It can be seen in cases of meandering pulmonary vein.

(d) Although lung hypoplasia and dextroposition of the heart are helpful signs, they can obscure the demonstration of the scimitar vein.

Question 3. Regarding imaging of scimitar syndrome:

(a) Chest radiography depicts all components of the syndrome, including the scimitar vein.

(b) Cardiac catheterisation and angiography are the preferred modalities for establishing the diagnosis.

(c) Conventional angiography is helpful in the evaluation of patients with high-grade pulmonary stenosis.

(d) Air trapping in the horseshoe lung segment should alert the physician to the possibility of bronchial stenosis.

Question 4. Regarding horseshoe lung:

(a) Horseshoe lung commonly occurs in association with scimitar syndrome.

(b) The isthmus lies posterior to the pericardial sac, oesophagus and aorta.

(c) Unilateral pulmonary hypoplasia is a constant feature of horseshoe lung.

(d) The bronchovascular supply to the isthmus is almost always from the normal lung.

Question 5. Preoperative imaging for scimitar syndrome should evaluate for:

(a) Total or partial pulmonary anomalous return.

(b) Shunt fraction.

(c) The presence of pulmonary hypertension.

(d) The presence of bronchopulmonary collaterals.

True

False

$\square$

$\square$

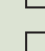

Doctor's particulars:

Name in full

MCR number

Email address

\section{SUBMISSION INSTRUCTIONS:}

(1) Visit the SMJ website: http://www.smj.org.sg/current-issue and select the appropriate set of questions. (2) Provide your name, email address and MCR number. (3) Select your answers and click "Submit".

\section{RESULTS:}

(1) Answers will be published online in the SMJ March 2017 issue. (2) The MCR numbers of successful candidates will be posted online at the SMJ website by 3 March 2017. (3) Passing mark is $60 \%$. No mark will be deducted for incorrect answers. (4) The SMJ editorial office will submit the list of successful candidates to the Singapore Medical Council. (5) One CME point is awarded for successful candidates.

Deadline for submission: (January 2017 SMJ 3B CME programme): 12 noon, 24 February 2017. 